\title{
ON MODEL MUTATION FOR REDUCTIVE CARTAN GEOMETRIES AND NON-EXISTENCE OF CARTAN SPACE FORMS
}

\author{
Antonio LotTA
}

\begin{abstract}
Reductive models $((\mathfrak{g}, \mathfrak{h}), H)$ for Cartan geometries are showed to fall into two classes, symmetric and non symmetric type, according to the existence or non existence of a mutation $\mathfrak{g}^{\prime}=\mathfrak{h} \oplus \mathfrak{m}$ where the $H$-module $\mathrm{m}$ is an abelian subalgebra. Sasakian structures are showed to be Cartan geometries having a model of non symmetric type and other examples of models of this type are exhibited. Reductive models for which no Cartan space forms exist are constructed. The phenomenon of non-existence of Cartan space forms pertains to models of non symmetric type.
\end{abstract}

\section{Introduction}

The notion of Cartan geometry originates from Élie Cartan's concept of "espace géneralisé". In Cartan's approach, a geometric structure on a manifold is regarded as a "deformation" of a standard model, which is always a homogeneous space. For example, a Riemannian manifold can be considered as a deformation (a non-homogeneous version) of the ordinary flat Euclidean space. So Cartan's theory appears as a natural generalization of Klein's Erlangen program. From this point of view a new concept of curvature arises (more general than the classic one), which measures how much the manifold under consideration differs from the standard model.

After Cartan, these ideas have been rigorously founded by C. Ehresmann [3] and S. Kobayashi ([4], [5]), and developed further by several authors.

In his monograph [12], R. W. Sharpe investigated some general aspects of Cartan geometries. One of the features of Sharpe's treatment is that the standard model of a Cartan geometry is not necessarily a homogeneous space $G / H$, but more generally is given by the following data of local nature:

i) An infinitesimal effective Klein pair $(\mathfrak{g}, \mathfrak{h})$;

ii) A Lie group $H$ with Lie algebra $\mathfrak{h}$;

2000 Mathematics Subject Classification: 53C05; 53B15.

Keywords and phrases: Cartan geometry, Cartan space form, reductive model for a Cartan geometry.

Received November 13, 2003; revised February 3, 2004. 
iii) A representation $A d: H \rightarrow A u t(\mathfrak{g})$, which extends the adjoint representation $H \rightarrow \operatorname{Aut}(\mathfrak{h})$.

Let $M$ be a real $n$-dimensional differentiable manifold and assume that $n=\operatorname{dim}_{\boldsymbol{R}} \mathfrak{g} / \mathfrak{h}$. Then, by definition, a Cartan geometry on $M$ modeled on $((\mathfrak{g}, \mathfrak{h}), H, A d)$ is a pair $(F, \omega)$ consisting of an $H$-principal bundle $F$ and a Cartan connection $\omega: T F \rightarrow \mathfrak{g}$ on it. For more details we refer the reader to section 2.

A relevant kind of Cartan geometries are the Cartan space forms. The latter generalize the space forms of Riemannian geometry in a natural manner. One of the most important results in [12] states that all Cartan space forms are locally Klein geometries, up to model mutation (cf. Theorem 2.3). Mutation is an alteration of the model which may change the curvature without losing information about the original geometry. The precise definition of model mutation is rehearsed in section 2.

Among Cartan geometries, the simplest are the reductive ones: these are characterized by the existence of a decomposition

$$
\mathfrak{g}=\mathfrak{h} \oplus \mathfrak{m}
$$

of the Lie algebra $\mathfrak{g}$, such that $\operatorname{Ad}(H) \mathfrak{m}=\mathfrak{m}$. This condition implies that the $\mathfrak{h}$ component of the Cartan connection $\omega$ is a principal connection on $F$, and the manifold $M$ can be endowed in a natural way with a linear connection. Such a linear connection depends on the choice of a reductive decomposition (1), see Lemma 3.2. Actually, if the representation $A d_{\mathfrak{m}}: H \rightarrow G L(\mathrm{~m})$ induced by $A d$ is faithful, a Cartan geometry on $M$ is equivalent to an $A d_{\mathfrak{m}}(H)$-structure and a linear connection on it (cf. [12], App. A or [1]).

About model mutation in the reductive case, the following result is given in [12], p. 220 (see also [13], Remark 4.6):

Lemma. Let $(\mathfrak{g}, \mathfrak{h})$ be a reductive model pair with group $H$. Write the $H$ module decomposition as $\mathfrak{g}=\mathfrak{h} \oplus \mathfrak{p}$. Then, there is, up to isomorphism, a unique mutant $\left(\mathfrak{g}^{\prime}, \mathfrak{h}\right)$ with $\mathfrak{g}^{\prime}=\mathfrak{h} \oplus \mathfrak{p}^{\prime}$ and $\left[\mathfrak{p}^{\prime}, \mathfrak{p}^{\prime}\right]=0$.

Hence, Sharpe's conclusion is that "for a reductive Cartan geometry we exclude nothing if we assume that $\left[\mathfrak{p}^{\prime}, \mathfrak{p}^{\prime}\right]=0$. This allows us to completely forget the large Lie algebra $\mathfrak{g}$ and retain only the subalgebra $\mathfrak{h}$ and the $H$-module p".

In this paper we examine carefully this point about mutation of reductive models. Namely, we show that there do exist reductive models for which the Lie bracket $\mathfrak{p} \times \mathfrak{p} \rightarrow \mathfrak{g}$ is essential, i.e. they do not admit any mutation $\left(\mathfrak{g}^{\prime}, \mathfrak{h}\right)$ such that $\left[\mathfrak{p}^{\prime}, \mathfrak{p}^{\prime}\right]=0$. This is the content of section 4 .

The reductive models having this feature will be called of non symmetric type, while those for which Sharpe's lemma is true will be called of symmetric type. This terminology is motivated by Proposition 4.1.

As a relevant example of Cartan geometries having a model of non symmetric type, we discuss $C R$-integrable contact metric structures, which include 
Sasakian structures. See Example 2 in $\$ 4$. Moreover, we show that any compact semisimple Lie algebra $q$ naturally gives rise to a model of non symmetric type by choosing as $H$ any Lie subgroup of $\operatorname{Aut}(\mathfrak{q})$ (Theorem 4.4).

In section 5 we prove that a model of non symmetric type is characterized by the property that for any torsion free Cartan geometry modeled on it, the linear connection induced on the base manifold $M$ by any reductive decomposition $\mathfrak{g}=\mathfrak{h} \oplus \mathfrak{m}$ has non vanishing torsion.

In the last paper of the paper we show that there are reductive models for which no Cartan space forms exist and that this can happen in any dimension $n \geq 3$ (cf. Corollaries 6.3 and 6.4).

This phenomenon is typical of models of non symmetric type since we prove in Theorem 6.1 that a necessary condition for a model to be of symmetric type is that there exists a Cartan space form modeled on it. In Theorem 6.2, we prove that for a class of first-order models, this condition is also sufficient.

\section{Cartan geometries}

In this section we collect the basic terminology about Cartan geometries following [12]. For this material, see also [13], [8] or [1].

An infinitesimal Klein pair is a pair $(\mathfrak{g}, \mathfrak{h})$ consisting of a finite-dimensional Lie algebra $\mathfrak{g}$ and a subalgebra $\mathfrak{h}$ of $\mathfrak{g}$. $(\mathfrak{g}, \mathfrak{h})$ is called effective if $\mathfrak{h}$ does not contain any non-trivial ideal of $\mathfrak{g}$.

A model for a Cartan geometry (cf. [12], p. 174), consists of a triple $((\mathfrak{g}, \mathfrak{h})$, $H, A d)$, denoted by $\xi_{H}^{A d}(\mathfrak{g}, \mathfrak{h})$, where $(\mathfrak{g}, \mathfrak{h})$ is an infinitesimal effective Klein pair $(\mathfrak{g}, \mathfrak{h}), H$ is a Lie group with Lie algebra $\mathfrak{h}$ and $A d: H \rightarrow A u t(\mathfrak{g})$ is a smooth representation extending the adjoint representation $H \rightarrow A u t(\mathfrak{h})$.

The group $H$ is called the structure group of the model. When the representation $A d$ is faithful, $\xi_{H}^{A d}(\mathfrak{g}, \mathfrak{h})$ is called effective. The dimension of $\xi_{H}^{A d}(\mathfrak{g}, \mathfrak{h})$ is $\operatorname{dim}_{\boldsymbol{R}} \mathfrak{g} / \mathfrak{h}$.

To each homogeneous space $G / H$ there is associated in a canonical way a model for a Cartan geometry with $\mathfrak{g}=\operatorname{Lie}(G), \mathfrak{h}=\operatorname{Lie}(H) ; \operatorname{Ad}: H \rightarrow \operatorname{Aut}(\mathfrak{g})$ is the restriction to $H$ of the adjoint representation of $G$.

Fix a model for a Cartan geometry $\xi_{H}^{A d}(\mathfrak{g}, \mathfrak{h})$, and let $M$ be a real differentiable manifold such that $\operatorname{dim}_{R} M=\operatorname{dim}_{R} \mathfrak{g} / \mathfrak{h}$.

A Cartan geometry on $M$ modeled on $\xi_{H}^{A d}(\mathfrak{g}, \mathfrak{h})$ is a pair $(F, \omega)$ consisting of an $H$-principal bundle $F$ and a Cartan connection $\omega$ on it with model $\xi_{H}^{A d}(\mathfrak{g}, \mathfrak{h})$. We shall also refer to the triple $(M,(F, \omega))$ as a Cartan geometry modeled on $\xi_{H}^{A d}(\mathfrak{g}, \mathfrak{h})$. We recall that, by definition, $\omega$ is an $H$-invariant smooth $\mathfrak{g}$-valued one form on $F$ which reproduces the fundamental vertical vector fields $A^{*}, A \in \mathfrak{h}$, and such that for each $u \in F, \omega_{u}: T_{u} F \rightarrow \mathfrak{g}$ is a linear isomorphism.

Let $\left(M_{1},\left(F_{1}, \omega_{1}\right)\right)$ and $\left(M_{2},\left(F_{2}, \omega_{2}\right)\right)$ be two Cartan geometries having the same model $\xi_{H}^{A d}(\mathfrak{g}, \mathfrak{h})$. A diffeomorphism $f: M_{1} \rightarrow M_{2}$ is called a geometric isomorphism between $\left(F_{1}, \omega_{1}\right)$ and $\left(F_{2}, \omega_{2}\right)$, if there exists a bundle isomorphism $b: F_{1} \rightarrow F_{2}$ covering $f$, such that $b^{*}\left(\omega_{2}\right)=\omega_{1}$. 
A Cartan geometry is called:

- effective if the corresponding model is effective.

- first-order if $A d_{\mathfrak{g} / \mathfrak{h}}: H \rightarrow G L(\mathfrak{g} / \mathfrak{h})$ is injective.

- connected if $F$ is connected.

- complete if the $\omega$-constant vector fields on $F$ are all complete.

- reductive if $\mathfrak{g}$ admits an $A d(H)$-module decomposition

$$
\mathfrak{g}=\mathfrak{h} \oplus \mathfrak{m} \text {. }
$$

A Cartan geometry on a manifold $M$ can be constructed from local data, called Cartan gauges. Let $M$ be a smooth manifold and let $\xi_{H}^{A d}(\mathfrak{g}, \mathfrak{h})$ be a model for a Cartan geometry. A (Cartan) gauge over $M$ modeled on $\xi_{H}^{A d}(\mathfrak{g}, \mathfrak{h})$ is a pair $(U, \theta)$ where $U$ is an open subset of $M$, and $\theta$ is a $\mathfrak{g}$-valued 1 -form defined on $U$, such that for each $x \in U$, the composition $p \circ \theta_{x}: T_{x} U \rightarrow \mathfrak{g} / \mathfrak{h}$ is a linear isomorphism. Here $p: \mathfrak{g} \rightarrow \mathfrak{g} / \mathfrak{h}$ is the canonical map.

Proposition 2.1. Let $M$ be a manifold. Let $\mathscr{C}_{M}$ be the set of Cartan connections on the trivial bundle $M \times H$ over $M$, modeled on $\xi_{H}^{A d}(\mathfrak{g}, \mathfrak{h})$. There is a bijective correspondence between $\mathscr{C}_{M}$ and the set $\mathscr{G}_{M}$ consisting of all Cartan gauges $(M, \theta)$. Each $\theta \in \mathscr{G}_{M}$ determines the Cartan connection $\omega_{\theta} \in \mathscr{C}_{M}$ defined by

$$
\omega_{\theta}(x, h)=\operatorname{Ad}\left(h^{-1}\right) \theta_{x}+\omega_{H}
$$

When $\omega_{H}$ is the Maurer-Cartan form of $H$.

Let $\xi_{H}^{A d}(\mathfrak{g}, \mathfrak{h})$ be a model for a Cartan geometry. A Cartan atlas on a manifold $M$ is a collection $\left\{\left(U_{\alpha}, \theta_{\alpha}\right)\right\}$ of gauges with the following properties:

1) The $U_{\alpha}$ 's form a covering of $M$;

2) For each $\alpha, \beta$, there exists a smooth map $k: U_{\alpha} \cap U_{\beta} \rightarrow H$, such that $\theta_{\beta}=\operatorname{Ad}\left(k^{-1}\right) \theta_{\alpha}+k^{*} \omega_{H}$.

Two Cartan atlases are called equivalent if their union is also a Cartan atlas; a Cartan structure is an equivalence class of Cartan atlases on $M$.

To each Cartan geometry $(M,(F, \omega))$ there is associated a Cartan structure on the base manifold $M$ in a canonical manner: a representative atlas consists of the gauges of the form $\theta_{\alpha}=\alpha^{*} \omega$, where $\alpha: U \rightarrow F$ is a local section of $F$. Any gauge $(U, \theta)$ belonging to this structure is called an admissible gauge. Conversely, when the model $\xi_{H}^{A d}(\mathfrak{g}, \mathfrak{h})$ is effective, a unique (up to gauge equivalence) Cartan geometry can be reconstructed from a given Cartan structure on a manifold $M$ (see [12]). Gauge equivalence means that $I d: M \rightarrow M$ is a geometrical isomorphism.

Next we recall the concept of curvature. Let $(F, \omega)$ be a Cartan geometry on a manifold $M$ with model $\xi_{H}^{A d}(\mathfrak{g}, \mathfrak{h})$. The curvature of $(F, \omega)$ is the $\mathfrak{g}$-valued 2-form $\Omega_{\omega}$ on $F$ defined by $\Omega_{\omega}:=d \omega+(1 / 2)[\omega, \omega]$. A Cartan geometry $(F, \omega)$ is called flat if $\Omega_{\omega}=0$; it is called torsion free if $\Omega_{\omega}$ takes values in $\mathfrak{h}$.

The curvature function of $(F, \omega)$ is a smooth map

$$
K: F \rightarrow C^{2}(\mathfrak{g} / \mathfrak{h}, \mathfrak{g})
$$

where $C^{2}(\mathfrak{g} / \mathfrak{h}, \mathfrak{g})$ is the linear space of all the bilinear alternating maps $\varphi: \mathfrak{g} / \mathfrak{h} \times$ 
$\mathfrak{g} / \mathfrak{h} \rightarrow \mathfrak{g} . \quad K$ is defined as follows: let $\tilde{X}, \tilde{Y} \in \mathfrak{g} / \mathfrak{h}$, and let $X, Y \in \mathfrak{g}$ be such that $p(X)=\tilde{X}, p(Y)=\tilde{Y}$. Then we set

$$
K(u)(\tilde{X}, \tilde{Y}):=\Omega_{u}\left(\omega_{u}^{-1} X, \omega_{u}^{-1} Y\right) .
$$

The definition is well-posed because the curvature form $\Omega$ of a Cartan connection is horizontal; this means that for each $u \in P$, and $\xi, \eta \in T_{u} F$, we have $\Omega_{u}(\xi, \eta)=0$ whenever $\xi$ or $\eta$ is vertical, i.e. tangent to the fiber of $F$ containing $u$ (see [12], Corollary 3.10 , p. 187).

Given an admissible gauge $\theta$, one can consider the local versions $\Omega^{\theta} \in$ $\Lambda^{2}(U, \mathfrak{g})$ and $K^{\theta}: U \rightarrow C^{2}(\mathfrak{g} / \mathfrak{h}, \mathfrak{g})$ of $\Omega$ and $K$ : they are defined by

$$
\Omega^{\theta}:=d \theta+\frac{1}{2}[\theta, \theta], \quad K^{\theta}(x)(\tilde{X}, \tilde{Y}):=\Omega_{x}^{\theta}\left(\left(p \theta_{x}\right)^{-1} \tilde{X},\left(p \theta_{x}\right)^{-1} \tilde{Y}\right), \quad x \in U .
$$

We remark that, when the geometry is reductive, with a fixed reductive decomposition $\mathfrak{g}=\mathfrak{h} \oplus \mathfrak{m}$, then $K$ and $K^{\theta}$ can be interpreted as maps taking values in $C^{2}(\mathfrak{m}, \mathfrak{g})$.

The structure group $H$ acts on $C^{2}(\mathfrak{g} / \mathfrak{h}, \mathfrak{g})$ by a representation $A d^{C}$ induced by $A d$ in a natural manner. We denote by $C_{H}^{2}(\mathfrak{g} / \mathfrak{h}, \mathfrak{g})$ the subset of $C^{2}(\mathfrak{g} / \mathfrak{h}, \mathfrak{g})$ consisting of all $A d^{C}(H)$-invariant elements. The proof of the following lemma is standard:

Lemma 2.2. Consider a Cartan geometry $(M,(F, \omega))$ with model $\xi_{H}^{A d}(\mathfrak{g}, \mathfrak{h})$ and the corresponding Cartan structure $\mathscr{C}$. Let $K_{0} \in C^{2}(\mathfrak{g} / \mathfrak{h}, \mathfrak{g})$ be fixed. Then the following are equivalent:

a) $(F, \omega)$ has constant curvature $K_{0}$;

b) $K_{0}$ belongs to $C_{H}^{2}(\mathfrak{g} / \mathfrak{h}, \mathfrak{g})$, and for each point $x \in M$ we can find a gauge $\theta$ of $\mathscr{C}$ at $x$, such that $K^{\theta}(x)=K_{0}$.

c) For every gauge $\theta$ of the Cartan structure $\mathscr{C}$ we have $K^{\theta}=$ const $=K_{0}$.

A Cartan space form modeled on $\xi_{H}^{A d}(\mathfrak{g}, \mathfrak{h})$ is a connected, complete, torsion free, geometrically oriented Cartan geometry whose curvature function is constant.

Geometrically oriented means that each $h \in H$ preserves the orientation in the sense that, fixed $u_{0} \in F$, there exists a path $\lambda$ in $F$ from $u_{0}$ to $u_{0} h$ whose development $\tilde{\lambda}$ via $a d \omega: T F \rightarrow \operatorname{End}(\mathfrak{g})$ ([12], p. 120) is a path joining $I d$ and $\operatorname{Ad}(h)$ in $\operatorname{Aut}(\mathfrak{g})$. For a connected geometry, this condition does not depend on the choice of $u_{0}$ ([12], p. 204).

Cartan space forms generalize in a natural manner the space forms of Riemannian geometry. One of the main results of this paper states the non existence of Cartan space forms for a class of reductive models $\xi_{H}^{A d}(\mathfrak{g}, \mathfrak{h})$.

Let $\xi_{H}^{A d}(\mathfrak{g}, \mathfrak{h})$ and $\xi_{H}^{A d^{\prime}}\left(\mathfrak{g}^{\prime}, \mathfrak{h}\right)$ be two models for a Cartan geometry with the same structure group $H$. Then $\xi_{H}^{A d^{\prime}}\left(\mathfrak{g}^{\prime}, \mathfrak{h}\right)$ is called a mutation of $\xi_{H}^{A d}(\mathfrak{g}, \mathfrak{h})$ if there exists a map $\lambda: \mathfrak{g} \rightarrow \mathfrak{g}^{\prime}$ which is an isomorphism between the $\operatorname{Ad}(H)$ module $\mathfrak{g}$ and the $A d^{\prime}(H)$-module $\mathfrak{g}^{\prime}$ satisfying the following conditions: 
i) $\lambda_{\mathfrak{l}}=i d_{\mathfrak{h}}$

ii) $[\lambda(X), \lambda(Y)]-\lambda[X, Y] \in \mathfrak{h}$ for all $X, Y \in \mathfrak{g}$.

Such a map $\lambda$ is called a mutation map.

It is proved in [12], p. 218, that given a Cartan geometry $\mathscr{C}=(M,(F, \omega))$ modeled on $\xi_{H}^{A d}(\mathfrak{g}, \mathfrak{h})$, and given a mutation $\xi_{H}^{A d^{\prime}}\left(\mathfrak{g}^{\prime}, \mathfrak{h}\right)$ of $\xi_{H}^{A d}(\mathfrak{g}, \mathfrak{h})$, with mutation map $\lambda: \mathfrak{g} \rightarrow \mathfrak{g}^{\prime}$, then $\mathscr{C}^{\prime}=(M,(F, \lambda \omega))$ is a Cartan geometry modeled on $\xi_{H}^{A d^{\prime}}\left(\mathfrak{g}^{\prime}, \mathfrak{h}\right)$. Moreover, if $\mathscr{C}$ is torsion free, $\mathscr{C}^{\prime}$ is also torsion free. We shall refer to $\mathscr{C}^{\prime}$ as a Cartan geometry obtained from $\mathscr{C}$ by mutation.

Mutation is a key notion involved in the classification of Cartan space forms. For the proof of the following result, see [12], Ch. 5.

Theorem 2.3. 1) Let $\mathscr{C}=(M,(F, \omega))$ be a flat Cartan space form modeled on $\xi_{H}^{A d}(\mathfrak{g}, \mathfrak{h})$. Then there is a connected Lie group $G$ with Lie algebra $\mathfrak{g}$, containing $H$ as a closed subgroup, and a subgroup $\Gamma \subset G$ such that $\left(\Gamma \backslash G, \omega_{\Gamma \backslash G}\right)$ is a locally Klein Cartan geometry on the double coset space $\Gamma \backslash G / H$ and such that $\mathscr{C}$ is geometrically isomorphic to it.

2) Let $\mathscr{C}=(M,(F, \omega))$ be a Cartan space form modeled on $\xi_{H}^{A d}(\mathfrak{g}, \mathfrak{h})$ and assume that either $H$ is connected or $M$ is simply connected. Then $\mathscr{C}$ is a mutation of a locally Klein geometry $\left(\Gamma \backslash G^{\prime}, \omega_{\Gamma \backslash G^{\prime}}\right)$ where $\xi_{H}^{A d^{\prime}}\left(\mathfrak{g}^{\prime}, \mathfrak{h}\right)$ is a suitable mutation of $\xi_{H}^{A d}(\mathfrak{g}, \mathfrak{h})$.

\section{Reductive models for a Cartan geometry}

Let $(F, \omega)$ be a reductive Cartan geometry on a manifold $M$ modeled on $\xi_{H}^{A d}(\mathfrak{g}, \mathfrak{h})$. Fix a reductive decomposition

$$
\mathfrak{g}=\mathfrak{h} \oplus \mathfrak{m}
$$

of $\mathfrak{g}$ and let $p r_{\mathfrak{m}}: \mathfrak{g} \rightarrow \mathfrak{m}, p r_{\mathfrak{h}}: \mathfrak{g} \rightarrow \mathfrak{h}$ be the linear projections determined by this decomposition. The Cartan connection $\omega$ determines in a natural manner a linear connection (covariant differentiation) $\nabla^{\mathfrak{m}}$ on $M$, depending on the choice of $\mathrm{m}$ (cf. [12], p. 197). $\nabla^{\mathfrak{m}}$ is determined locally as follows

$$
\theta_{\mathfrak{m}}\left(\nabla_{X}^{\mathfrak{m}} Y\right)=X\left(\theta_{\mathfrak{m}}(Y)\right)+\left[\theta_{\mathfrak{h}}(X), \theta_{\mathfrak{m}}(Y)\right], \quad X, Y \in \mathfrak{X}(U)
$$

where $(U, \theta)$ is an admissible gauge and $\theta_{\mathfrak{h}}: T U \rightarrow \mathfrak{h}, \theta_{\mathfrak{m}}: T U \rightarrow \mathfrak{m}$ are the 1forms on the open set $U$ given by $\theta_{\mathfrak{h}}=p r_{\mathfrak{h}} \circ \theta$ and $\theta_{\mathfrak{m}}=p r_{\mathfrak{m}} \circ \theta$. We recall that, according to the definition of gauge, $\theta_{\mathfrak{m}}(x): T_{x} M \rightarrow \mathfrak{m}$ is a linear isomorphism for each $x \in U$.

Lemma 3.1. Let $\xi_{H}^{A d}(\mathfrak{g}, \mathfrak{h})$ be a reductive model for a Cartan geometry. Fix a reductive decomposition $\mathfrak{g}=\mathfrak{h} \oplus \mathfrak{m}$. Let $(M,(F, \omega))$ be a Cartan geometry modeled on $\xi_{H}^{A d}(\mathfrak{g}, \mathfrak{h})$ and let $\nabla$ the corresponding linear connection with torsion $T$. Let $\theta: T U \rightarrow \mathfrak{g}$ be an admissible Cartan gauge at $x$. Then we have the following formula

$$
K_{\mathfrak{m}}^{\theta}(x)(Z, W)=\theta_{\mathfrak{m}}\left(T_{x}(X, Y)\right)+[Z, W]_{\mathfrak{m}}, \quad x \in U
$$

where $X, Y \in T_{x} M$ and $Z=\theta_{\mathfrak{m}}(X), W=\theta_{\mathfrak{m}}(Y)$. 
In particular, $\nabla$ has vanishing torsion provided the Cartan geometry is torsion free and $[\mathrm{m}, \mathrm{m}]=0$.

Proof. This follows by a straightforward computation using (2).

Lemma 3.2. Let $\xi_{H}^{A d}(\mathfrak{g}, \mathfrak{h})$ be a reductive model for a Cartan geometry and let $\xi_{H}^{A d^{\prime}}\left(\mathfrak{g}^{\prime}, \mathfrak{h}\right)$ be a mutation of $\xi_{H}^{A d}(\mathfrak{g}, \mathfrak{h})$ with mutation map $\lambda: \mathfrak{g} \rightarrow \mathfrak{g}^{\prime}$. Let

$$
\mathfrak{g}=\mathfrak{h} \oplus \mathfrak{m}, \quad \mathfrak{g}^{\prime}=\mathfrak{h} \oplus \mathfrak{m}^{\prime}
$$

be reductive decompositions of $\mathfrak{g}$ and $\mathfrak{g}^{\prime}$. Let $(M,(F, \omega))$ be a Cartan geometry modeled on $\xi_{H}^{A d}(\mathfrak{g}, \mathfrak{h})$, and let $\mathscr{C}^{\prime}=(M,(F, \lambda \omega))$ be the Cartan geometry obtained by mutation.

The linear connections $\nabla$ and $\nabla^{\prime}$ induced by the Cartan geometries $\mathscr{C}$ resp. $\mathscr{C}^{\prime}$ via the reductive decompositions (4) are related by

$$
\nabla_{X}^{\prime} Y=\nabla_{X} Y+\theta_{\mathfrak{m}}^{-1}\left[\phi \theta_{\mathfrak{m}}(X), \theta_{\mathfrak{m}}(Y)\right], \quad X, Y \in \mathfrak{X}(U)
$$

where $\theta: T U \rightarrow \mathfrak{g}$ is an admissible gauge for $\mathscr{C},[$,$] is the Lie bracket of \mathfrak{g}$, and

$$
\phi: \mathfrak{m} \rightarrow \mathfrak{h}
$$

is the linear map defined by the requirement that

$$
\phi(Z)-\lambda(Z) \in \mathfrak{m}^{\prime} \quad \forall Z \in \mathfrak{m} .
$$

Proof. We denote by $p r_{\mathfrak{m}^{\prime}}^{\prime}: \mathfrak{g}^{\prime} \rightarrow \mathfrak{m}^{\prime}$ and $p r_{\mathfrak{h}}^{\prime}: \mathfrak{g}^{\prime} \rightarrow \mathfrak{h}$ the linear projections determined by the decomposition $\mathfrak{g}^{\prime}=\mathfrak{h} \oplus \mathfrak{m}^{\prime}$. Hence by definition $\phi=p r_{\mathfrak{h}}^{\prime} \circ \lambda$, and the composition $\psi:=p r_{\mathfrak{m}^{\prime}}^{\prime} \circ \lambda: \mathfrak{m} \rightarrow \mathfrak{m}^{\prime}$ is a linear isomorphism. According to the definition of mutation, we see that

$$
\psi[H, Z]=[H, \psi Z]^{\prime}, \quad H \in \mathfrak{h}, Z \in \mathfrak{m} .
$$

Moreover we have that $\theta^{\prime}:=\lambda \circ \theta: T U \rightarrow \mathfrak{g}^{\prime}$ is an admissible gauge for $\mathscr{C}^{\prime}$, such that

$$
\theta_{\mathfrak{m}^{\prime}}^{\prime}:=p r_{\mathfrak{m}^{\prime}}^{\prime} \circ \theta^{\prime}=\psi \theta_{\mathfrak{m}}, \quad \theta_{\mathfrak{h}}^{\prime}:=p r_{\mathfrak{h}}^{\prime} \circ \theta^{\prime}=\theta_{\mathfrak{h}}+\phi \theta_{\mathfrak{m}} .
$$

Hence taking into account the expression of $\nabla_{X}^{\prime} Y$ in the gauge $\theta^{\prime}$, we get

$$
\begin{aligned}
\theta_{\mathfrak{m}^{\prime}}^{\prime}\left(\nabla_{X}^{\prime} Y\right) & =X\left(\theta_{\mathfrak{m}^{\prime}}^{\prime}(Y)\right)+\left[\theta_{\mathfrak{h}}^{\prime}(X), \theta_{\mathfrak{m}^{\prime}}^{\prime}(Y)\right]^{\prime} \\
& =X\left(\psi \theta_{\mathfrak{m}}(Y)\right)+\left[\theta_{\mathfrak{h}}(X), \psi \theta_{\mathfrak{m}}(Y)\right]^{\prime}+\left[\phi \theta_{\mathfrak{m}}(X), \psi \theta_{\mathfrak{m}}(Y)\right]^{\prime}
\end{aligned}
$$

whence, using (6):

$$
\psi \theta_{\mathfrak{m}}\left(\nabla_{X}^{\prime} Y\right)=X\left(\psi \theta_{\mathfrak{m}}(Y)\right)+\psi\left[\theta_{\mathfrak{h}}(X), \theta_{\mathfrak{m}}(Y)\right]+\psi\left[\phi \theta_{\mathfrak{m}}(X), \theta_{\mathfrak{m}}(Y)\right]
$$

which yields

$$
\theta_{\mathfrak{m}}\left(\nabla_{X}^{\prime} Y\right)=\theta_{\mathfrak{m}}\left(\nabla_{X} Y\right)+\left[\phi \theta_{\mathfrak{m}}(X), \theta_{\mathfrak{m}}(Y)\right]
$$

and the assertion follows. 


\section{Reductive models of non symmetric type}

The following result should be compared with Lemma 6.4 of [12], page 220, and its proof.

Proposition 4.1. Let $\xi_{H}^{A d}(\mathfrak{g}, \mathfrak{h})$ be a reductive model for a Cartan geometry with group $H$. The following conditions are equivalent:

a) there exists a mutation $\xi_{H}^{A d^{\prime}}\left(\mathfrak{g}^{\prime}, \mathfrak{h}\right)$ of $\xi_{H}^{A d}(\mathfrak{g}, \mathfrak{h})$ admitting a reductive decomposition $\mathfrak{g}^{\prime}=\mathfrak{h} \oplus \mathfrak{m}^{\prime}$ such that $\left[\mathfrak{m}^{\prime}, \mathfrak{m}^{\prime}\right]^{\prime}=0$;

b) $\mathfrak{g}$ admits a reductive decomposition $\mathfrak{g}=\mathfrak{h} \oplus \mathfrak{m}$ such that $[\mathfrak{m}, \mathfrak{m}] \subset \mathfrak{h}$.

When these conditions are satisfied the mutation of $\xi_{H}^{A d}(\mathfrak{g}, \mathfrak{h})$ satisfying a) is unique up to isomorphism and is obtained from a reductive decomposition of $\mathfrak{g}$ like in $\mathrm{b}$ ) setting $\mathfrak{g}^{\prime}=\mathfrak{g}$ with $\mathfrak{m}^{\prime}=\mathfrak{m}$ as an $\mathfrak{h}$-module and Lie bracket on $\mathfrak{m}^{\prime}$ defined by $[\mathrm{m}, \mathrm{m}]^{\prime}=0$.

Proof. Assuming a), if $\lambda: \mathfrak{g} \rightarrow \mathfrak{g}^{\prime}$ is the corresponding mutation map, it suffices to set $\mathfrak{m}=\lambda^{-1}\left(\mathfrak{m}^{\prime}\right)$ to obtain a reductive decomposition of $\mathfrak{g}$ like in $b$ ). The converse is proved constructing the Lie algebra $\mathfrak{g}^{\prime}$ as indicated in the last part of the assertion. The mutation map $\mathfrak{g} \rightarrow \mathfrak{g}^{\prime}$ is the canonical map.

This observation leads to the following

Definition 4.2. Let $\xi_{H}^{A d}(\mathfrak{g}, \mathfrak{h})$ be a reductive model for a Cartan geometry with group $H$. We shall say that $\xi_{H}^{A d}(\mathfrak{g}, \mathfrak{h})$ is of symmetric type if there exists a mutation $\xi_{H}^{A d^{\prime}}\left(\mathfrak{g}^{\prime}, \mathfrak{h}\right)$ of $\xi_{H}^{A d}(\mathfrak{g}, \mathfrak{h})$ admitting a reductive decomposition $\mathfrak{g}^{\prime}=\mathfrak{h} \oplus \mathfrak{m}^{\prime}$ such that $\left[\mathfrak{m}^{\prime}, \mathfrak{m}^{\prime}\right]^{\prime}=0$. Otherwise $\xi_{H}^{A d}(\mathfrak{g}, \mathfrak{h})$ will be called of non symmetric type.

According to Proposition 4.1, we see that reductive models of non symmetric type exist and arise in a natural way. For example:

COROllary 4.3. 1) Let $(M, g)$ be a homogeneous Riemannian manifold which is not locally Riemannian symmetric. Let $G$ be any Lie group acting transitively on $M$ as a group of isometries with isotropy subgroup $H$ at a point $x_{o} \in M$. Then $G / H$ is a reductive model for a Cartan geometry which is of non symmetric type.

2) Let $(M, g)$ be a complete, simply connected Riemannian manifold. Then $M$ is Riemannian symmetric if and only if there exists a transitive group of isometries $G \subset I_{g}(M)$ such that $G / H$ is a reductive model for a Cartan geometry of symmetric type, where $H$ is the isotropy subgroup at some point of $M$.

In the following we describe other examples of models of non symmetric type, which are constructed starting with a Lie algebra $q$ choosing as structure group a Lie subgroup $H$ of the automorphism group $\operatorname{Aut}(\mathfrak{q})$.

Set $\mathfrak{g}:=\mathfrak{h} \oplus \mathfrak{q}$, where $\mathfrak{h}$ is the Lie algebra of $H$. The vector space $\mathfrak{g}$ has a 
natural structure of a Lie algebra, admitting $\mathfrak{q}$ and $\mathfrak{h}$ as Lie subalgebras, and such that

$$
[A, X]:=A(X)=-[X, A]
$$

for all $X \in \mathfrak{q}$ and $A \in \mathfrak{h}$. Then $\xi_{H}^{A d}(\mathfrak{g}, \mathfrak{h})$ is a reductive model for a Cartan geometry with group $H$, where $A d: H \rightarrow A u t(\mathfrak{g})$ is the representation such that

$$
\operatorname{Ad}(h)(X+A)=h(X)+A d_{H}(A), \quad X \in \mathfrak{q}, A \in \mathfrak{h} .
$$

We shall refer to this model as the standard reductive model for a Cartan geometry with group $H$.

THEOREM 4.4. Let $\mathfrak{q}$ be a non abelian Lie algebra and let $H \subset \operatorname{Aut}(\mathfrak{q})$ be a Lie subgroup. In each of the following cases, the standard reductive model for a Cartan geometry with group $H$ is of non symmetric type:

1) $H$ is discrete;

2) q is compact semisimple;

3) There exists a vector subspace $V \subset \mathfrak{q}$ such that $[V, V] \not \subset V$ and $a(V) \subset V$ for each $a \in H$.

Proof. In each case we argue by contradiction. Assume that our model $\xi_{H}^{A d}(\mathfrak{g}, \mathfrak{h})$ is of symmetric type. We fix a reductive decomposition $\mathfrak{g}=\mathfrak{h} \oplus \mathfrak{m}$ such that $[\mathfrak{m}, \mathfrak{m}] \subset \mathfrak{h}$, and we consider the mutation $\xi_{H}^{A d^{\prime}}\left(\mathfrak{g}^{\prime}, \mathfrak{h}\right)$ of $\xi_{H}^{A d}(\mathfrak{g}, \mathfrak{h})$ satisfying the condition $b$ ) of Proposition 4.1. We recall that the mutation map $\lambda: \mathfrak{g} \rightarrow \mathfrak{g}^{\prime}$ is the identical map of $\mathfrak{g}$.

In case 1 ), being $\mathfrak{h}=0$, it follows that $\mathfrak{g}$ is abelian, which is absurd since $\mathfrak{q}$ is not abelian.

Now we consider case 2). As in Lemma 3.2 we consider the linear maps $\psi: \mathfrak{m} \rightarrow \mathfrak{m}^{\prime}$ and $\phi: \mathfrak{m} \rightarrow \mathfrak{h}$ such that $X=\psi(X)+\phi(X)$ for each $X \in \mathfrak{m}$.

We fix an $\operatorname{Aut}(\mathfrak{q})$-invariant scalar product $\langle$,$\rangle on \mathfrak{q}$ and we consider a compact Lie group $M$ with Lie algebra $\mathfrak{q}$. Then $\langle$,$\rangle determines a biinvariant$ Riemannian metric $g$ on $M$. The Maurer-Cartan form $\omega_{\mathfrak{q}}: T M \rightarrow \mathfrak{q}$ gives rises to a globally defined Cartan gauge

$$
\theta: T M \rightarrow \mathfrak{g}, \quad \theta=i \circ \omega_{\mathfrak{q}}, \quad i: \mathfrak{q} \hookrightarrow \mathfrak{g} \text { inclusion }
$$

modeled on $\xi_{H}^{A d}(\mathfrak{g}, \mathfrak{h})$. This gauge in turn determines a Cartan connection $\omega$ on the trivial bundle $P=M \times H \rightarrow M$. Clearly, according to the structure equation $d \omega_{\mathfrak{q}}+(1 / 2)\left[\omega_{\mathfrak{q}}, \omega_{\mathrm{q}}\right]=0$, the Cartan geometry $\mathscr{C}=(M,(P, \omega))$ is flat. According to (2), the linear connection $\nabla$ induced on $M$ associated with the reductive decomposition $\mathfrak{g}=\mathfrak{h} \oplus \mathfrak{q}$ is determined as follows

$$
\nabla_{X} Y=0 \quad \forall X, Y \in \mathfrak{q} .
$$

In particular, the metric $g$ is parallel with respect to $\nabla$.

As in Lemma 3.2, we consider the linear maps $\psi: \mathfrak{q} \rightarrow \mathrm{m}^{\prime}$ and $\phi: \mathfrak{q} \rightarrow \mathfrak{h}$ such that $X=\lambda(X)=\psi(X)+\phi(X)$ for each $X \in \mathfrak{q}$. By using (5) we see that $g$ is also parallel with respect to the linear connection $\nabla^{\prime}$ associated with the muted 
geometry $\left(P, \omega^{\prime}\right)$ via the decomposition $\mathfrak{g}^{\prime}=\mathfrak{h} \oplus \mathfrak{m}^{\prime}$. Indeed, this follows from the fact that for each $Z \in \mathfrak{h}, a d_{Z}$ is a skew-symmetric endomorphism of $\mathfrak{q}$. Since $\mathscr{C}$ is flat, $\mathscr{C}^{\prime}$ is torsion free. Hence according to Lemma 3.1 , since $\left[\mathrm{m}^{\prime}, \mathrm{m}^{\prime}\right]^{\prime}=0$, $\nabla^{\prime}$ has vanishing torsion. Thus $\nabla^{\prime}$ is the Levi-Civita connection of $g$. Since $g$ is biinvariant we have

$$
\nabla_{X}^{\prime} Y=\frac{1}{2}[X, Y] \quad \forall X, Y \in \mathfrak{q} .
$$

Substituting in (5) this yields

$$
\phi(X)=\frac{1}{2} a d_{X} \quad \forall X \in \mathfrak{q} .
$$

All derivations of $\mathfrak{q}$ being inner, this leads to the conclusion that $\phi: \mathfrak{q} \rightarrow \mathfrak{h}$ is onto. Hence we must have $\mathfrak{h}=0$, so that $H$ is discrete and this contradicts case 1).

Case 3). We consider again a Lie group $M$ with $\operatorname{Lie}(M)=\mathfrak{q}$ endowed with the Cartan geometries $\mathscr{C}$ obtained from the global gauge (7), its mutation $\mathscr{C}^{\prime}$ and the linear connections $\nabla$ and $\nabla^{\prime}$. Denote by $\mathscr{V}$ the left invariant smooth distribution on $M$ defined by

$$
\mathscr{V}_{x}=\left(\omega_{\mathfrak{q}}\right)_{x}^{-1}(V), \quad x \in M .
$$

We remark that, according to (8), $\mathscr{V}$ is parallel with respect to $\nabla$. Since $\nabla$ and $\nabla^{\prime}$ are related by (5) we see that $\mathscr{V}$ is also parallel with respect to $\nabla^{\prime}$. Indeed, this follows at once from the fact that $[\mathfrak{h}, V] \subset V$. Since $\nabla^{\prime}$ has vanishing torsion and $\mathscr{V}$ is $\nabla^{\prime}$-parallel we conclude that $\mathscr{V}$ is Frobenius integrable. We have arrived at a contradiction because by assumption $[V, V] \not \subset V$.

Examples. 1. (Complete parallelism) In the above result an admissible choice for $H$ is $H=\{i d\}$. A Cartan geometry $(M,(F, \omega))$ modeled on $\xi_{H}^{A d}(\mathfrak{g}, \mathfrak{h})$ is a complete parallelism on the base manifold $M$. Notice that the flat Cartan geometries modeled on $\xi_{H}^{A d}(\mathfrak{g}, \mathfrak{h})$ are the parallelizable manifolds $M$ such that the Lie algebra $\mathfrak{X}(M)$ is isomorphic to $\mathfrak{q}$.

2. (Contact metric structures) Using a result in [9], we shall exhibit a canonical interpretation of $\mathrm{CR}$-integrable contact metric manifolds as reductive Cartan geometries with non trivial structure group, whose model is of non symmetric type and is constructed according to Theorem 4.4. We refer the reader to [2], [10] or [15] for the notions of contact metric manifold, CR manifold and Sasakian manifold.

Consider the real vector space $V=\mathbf{R}^{2 k}$ where $k \geq 1$. We denote by $\left\{x_{1}, \ldots, x_{2 k}\right\}$ the standard basis and by $\langle$,$\rangle the standard inner product on V$. Moreover, let $J: V \rightarrow V$ be the complex structure associated to the matrix

$$
\left(\begin{array}{cc}
0 & -I_{k} \\
I_{k} & 0
\end{array}\right)
$$


with respect to the basis $\left\{x_{1}, \ldots, x_{2 k}\right\}$. We introduce a Lie algebra structure on $\mathfrak{q}:=V \oplus \boldsymbol{R}$ as follows. We set

$$
[x, y]:=-2\langle x, J y\rangle, \quad[x, t]=[t, x]=[s, t]:=0
$$

for all $x, y \in V$ and $s, t \in \boldsymbol{R}$.

The complex structure $J: V \rightarrow V$ extends to a derivation $\varphi: \mathfrak{q} \rightarrow \mathfrak{q}$ by setting $\varphi=0$ on $\boldsymbol{R}$. We also extend $\langle$,$\rangle to an inner product g$ on $\mathfrak{q}$ in such a way that

$$
g(x, y)=\langle x, y\rangle, \quad g(x, t)=0, \quad g(t, s)=s t .
$$

Then $(\mathfrak{q}, \varphi, g)$ is a metric $f$-structure with rank $2 k$ (see [11]); its structure group is the closed subgroup of $G L(\mathfrak{q})$ defined by

$$
H=\{a \in O(\mathfrak{q}, g) \mid a \circ \varphi=\varphi \circ a, a(1)=1\} \cong U(k) \times 1 .
$$

We remark that $H \subset A u t(\mathfrak{q})$ and that each $a \in H$ leaves $V$ invariant. The Lie algebra $\mathfrak{h}$ of $H$ consists of all derivations $D$ of $\mathfrak{q}$ which commute with $\varphi$, vanish on $\boldsymbol{R}$, and such that

$$
g(D z, w)+g(z, D w)=0 .
$$

Then according to the above theorem, case 3), the standard reductive model $\xi_{H}^{A d}(\mathfrak{g}, \mathfrak{h})$ for a Cartan geometry with group $H$ is of non symmetric type.

Cartan geometries modeled on $\xi_{H}^{A d}(\mathfrak{g}, \mathfrak{h})$ are linked with contact structures. Namely, denote by $\mathscr{M}$ the subspace of $C^{2}(\mathfrak{q}, \mathfrak{g})$ consisting of the bilinear maps $\psi: \mathfrak{q} \times \mathfrak{q} \rightarrow \mathfrak{g}$ such that

$$
\psi_{\mathfrak{q}}(x, y)=\psi_{\mathfrak{q}}(s, t)=0, \quad \psi_{\mathfrak{q}}(s, \varphi x)=-\varphi \psi_{\mathfrak{q}}(s, x)
$$

for all $x, y \in V$ and $s, t \in \boldsymbol{R}$. We quote the following

THeORem 4.5 [9]. Let $M$ be a real manifold of dimension $2 k+1$. There is a natural bijection between the set of CR-integrable contact metric structures on $M$ and the set of Cartan geometries on $M$ modeled on $\xi_{H}^{A d}(\mathfrak{g}, \mathfrak{h})$ and of curvature type $\mathscr{M}$, modulo gauge equivalence. Moreover, the Sasakian structures correspond to the torsion free Cartan geometries.

Here the expression "Cartan geometry of curvature type $\mathscr{M}$ " refers to a Cartan geometry whose curvature function $K$ takes values in the $H$-submodule $\mathscr{M}$ of $C^{2}(\mathfrak{q}, \mathfrak{g})$. Actually, the above result is proved in the more general context of almost $\mathscr{S}$-structures.

We remark that given a CR-integrable contact metric manifold, the linear connection induced by the corresponding Cartan geometry is the Tanaka-Webster connection (see e.g. [14] or [15]). This is a special connection with torsion adapted to the contact structure. The fact that this connection has non vanishing torsion is not accidental and comes from a general property of models of non symmetric type as showed in the next section. 


\section{Geometric characterization of non symmetric models}

THeOREM 5.1. Let $\xi_{H}^{A d}(\mathfrak{g}, \mathfrak{h})$ be a reductive model for a Cartan geometry. Then the following are equivalent:

a) $\xi_{H}^{A d}(\mathfrak{g}, \mathfrak{h})$ is of non symmetric type;

b) For every torsion free Cartan geometry $(M,(F, \omega))$ modeled on $\xi_{H}^{A d}(\mathfrak{g}, \mathfrak{h})$, the linear connection $\nabla^{\mathfrak{m}}$ on $M$ induced by any reductive decomposition $\mathfrak{g}=\mathfrak{h} \oplus \mathrm{m}$ has non vanishing torsion.

Proof. a $\Rightarrow$ b) Consider a torsion free Cartan geometry $(M,(F, \omega))$ modeled on $\xi_{H}^{A d}(\mathfrak{g}, \mathfrak{h})$ and choose a reductive decomposition $\mathfrak{g}=\mathfrak{h} \oplus \mathfrak{m}$. Assume by contradiction that $\nabla^{\mathfrak{m}}$ has vanishing torsion. Then by using formula (3) we obtain that $[\mathfrak{m}, \mathfrak{m}]_{\mathfrak{m}}=0$, whence $[\mathfrak{m}, \mathfrak{m}] \subset \mathfrak{h}$. According to Proposition 4.1 this implies that $\xi_{H}^{A d}(\mathfrak{g}, \mathfrak{h})$ is of symmetric type which is a contradiction.

b) $\Rightarrow$ a) Assume by contradiction that $\xi_{H}^{A d}(\mathfrak{g}, \mathfrak{h})$ is of symmetric type. Consider a reductive decomposition $\mathfrak{g}=\mathfrak{h} \oplus \mathfrak{m}$ with $[\mathfrak{m}, \mathfrak{m}] \subset \mathfrak{h}$ and the canonical mutation $\xi_{H}^{A d d^{\prime}}\left(\mathfrak{g}^{\prime}, \mathfrak{h}\right)$ of $\xi_{H}^{A d}(\mathfrak{g}, \mathfrak{h})$ according to Proposition 4.1. Consider the absolute parallelism

$$
\gamma: T \mathrm{~m} \rightarrow \mathrm{m}
$$

on the vector space $m$ endowed with the natural manifold structure. It gives rise to a globally defined Cartan gauge

$$
\theta: T \mathrm{~m} \rightarrow \mathfrak{g}, \quad \theta=i \circ \gamma, \quad i: \mathfrak{m} \hookrightarrow \mathfrak{g} \text { inclusion. }
$$

Hence $\theta$ determines a Cartan connection $\omega$ on the trivial $H$-principal bundle $P=\mathfrak{m} \times H \rightarrow \mathfrak{m}$, such that $\theta=\sigma^{*}(\omega)$, where $\sigma: \mathfrak{m} \rightarrow \mathfrak{m} \times H$ is the canonical section. A straightforward computation shows that the curvature function $K^{\theta}$ : $\mathrm{m} \rightarrow C^{2}(\mathrm{~m}, \mathfrak{g})$ is constant, namely

$$
K^{\theta}(x)(Z, W)=[Z, W], \quad x \in \mathfrak{m} .
$$

Hence according to $b)$ in Lemma 2.2 , the Cartan geometry $(m,(F, \omega))$ has constant curvature given by the Lie bracket $\mathfrak{m} \times \mathfrak{m} \rightarrow \mathfrak{g}$. In particular, this geometry is torsion free because $[\mathfrak{m}, \mathfrak{m}] \subset \mathfrak{h}$. On the other hand, according to (3.1), the linear connection $\nabla^{\mathrm{m}}$ on $\mathrm{m}$ determined by the reductive decomposition $\mathfrak{g}=\mathfrak{h} \oplus \mathfrak{m}$ has vanishing torsion, and this contradicts the assumption $\mathfrak{b}$ ).

\section{On the existence of Cartan space forms}

The aim of this section is to show that for a reductive model of non symmetric type the phenomenon of non existence of Cartan space forms modeled on it may occur.

First of all we prove the following

THEOREM 6.1. Let $\xi_{H}^{A d}(\mathfrak{g}, \mathfrak{h})$ be a reductive model for a Cartan geometry with group $H$. Assume that $H$ is connected. A necessary condition for $\xi_{H}^{A d}(\mathfrak{g}, \mathfrak{h})$ to be of symmetric type is that there exists a Cartan space form modeled on it. 
Proof. Assume $\xi_{H}^{A d}(\mathfrak{g}, \mathfrak{h})$ of symmetric type and consider a reductive decomposition $\mathfrak{g}=\mathfrak{h} \oplus \mathfrak{m}$ with $[\mathfrak{m}, \mathfrak{m}] \subset \mathfrak{h}$. In the proof of $b) \Rightarrow a$ ) of Theorem 5.1 we have seen that the vector space $m$ carries a torsion free Cartan geometry $(P, \omega)$ modeled on $\xi_{H}^{A d}(\mathfrak{g}, \mathfrak{h})$ with constant curvature given by the Lie bracket $\mathrm{m} \times \mathrm{m} \rightarrow \mathfrak{h}$. Actually, $(\mathrm{m},(P, \omega))$ is a Cartan space form. Indeed, $P=\mathfrak{m} \times H$ is connected whence $(P, \omega)$ is geometrically oriented. Moreover, $(P, \omega)$ is complete since a $\omega$-constant vector field $A$ on $P$ is of the form

$$
A_{(x, h)}=\left(\gamma_{x}^{-1}(A d(h) Z), Y_{h}\right), \quad(x, h) \in \mathfrak{m} \times H
$$

where $Z \in \mathrm{m}$ and $Y$ is a left-invariant vector field on $H$. Here $\gamma: T \mathrm{~m} \rightarrow \mathrm{m}$ is the absolute parallelism on $\mathrm{m}$. Hence the integral curve of $A$ with initial point $\left(x_{0}, h_{0}\right)$ is given by

$$
\psi(t)=\left(x_{0}+\int_{0}^{t} A d\left(h_{0} \exp \left(s Y_{e}\right)\right) Z d s, h_{0} \exp \left(t Y_{e}\right)\right) .
$$

Next we consider a case where the existence of a Cartan space form is also a sufficient condition for the model to be of symmetric type.

THeOREM 6.2. Let $\xi_{H}^{A d}(\mathfrak{g}, \mathfrak{h})$ be a first order reductive model for a Cartan geometry with connected group $H$. Fix a reductive decomposition $\mathfrak{g}=\mathfrak{h} \oplus \mathrm{m}$.

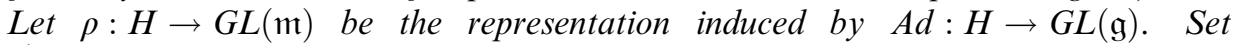
$\mathfrak{h}^{\prime}:=d \rho(\mathfrak{h})$. Let $\Phi: \mathfrak{m} \rightarrow \operatorname{End}(\mathfrak{m})$ be the linear map such that

$$
\forall X, Y \in \mathfrak{m} \quad \Phi(X)(Y)=[X, Y]_{\mathfrak{m}} .
$$

Assume that $\Phi(\mathfrak{m}) \subset \mathfrak{h}^{\prime}$.

Then the following are equivalent:

a) $\xi_{H}^{A d}(\mathfrak{g}, \mathfrak{h})$ is of symmetric type;

b) There exists a Cartan space form modeled on $\xi_{H}^{A d}(\mathfrak{g}, \mathfrak{h})$.

Proof. We have to show that $\mathrm{b}) \Rightarrow \mathrm{a})$. Let $M$ be a Cartan space form modeled on $\xi_{H}^{A d}(\mathfrak{g}, \mathfrak{h})$ with curvature $K$. We can consider $K$ as a bilinear map $\mathfrak{g} \times \mathfrak{g} \rightarrow \mathfrak{h}$ such that $K(\mathfrak{g}, \mathfrak{h})=K(\mathfrak{h}, \mathfrak{g})=0$.

According to Theorem 2.32 ), since $H$ is connected, we can assume that $K=0$ after a mutation of $\xi_{H}^{A d}(\mathfrak{g}, \mathfrak{h})$. More precisely, setting

$$
[X, Y]^{\prime}=[X, Y]-K(X, Y)
$$

we get a new Lie algebra structure on $\mathfrak{g}^{\prime}=\mathfrak{g}$, such that $\xi_{H}^{A d^{\prime}}\left(\mathfrak{g}^{\prime}, \mathfrak{h}\right)$ is a mutation of $\xi_{H}^{A d}(\mathfrak{g}, \mathfrak{h})$. With respect to $\xi_{H}^{A d^{\prime}}\left(\mathfrak{g}^{\prime}, \mathfrak{h}\right), M$ is a flat Cartan space form (cf. [12], p. 222-224). Notice that our assumption on $\Phi$ is still applicable to $\xi_{H}^{A d^{\prime}}\left(\mathfrak{g}^{\prime}, \mathfrak{h}\right)$, because the Lie bracket $\mathfrak{h} \times \mathfrak{m} \rightarrow \mathfrak{m}$ does not change after the mutation. Since also the property a) is invariant under mutation, to prove that b) $\Rightarrow$ a) we can assume at the outset that $M$ is a flat Cartan space form modeled on $\xi_{H}^{A d}(\mathfrak{g}, \mathfrak{h})$.

By using Theorem 2.31 ), we can construct a homogeneous space $G / H$ where $G$ is a Lie group with Lie algebra $\mathfrak{g}$, containing $H$ as a Lie subgroup. Clearly, $G / H$ is a reductive homogeneous space in the sense of [7], Ch. X. 
Since the model is of first order, the principal $H$-fiber bundle $\pi: G \rightarrow G / H$ imbeds in the bundle of linear frames $L(G / H)$ via the mapping

$$
\Psi: g \in G \mapsto \pi_{*} \circ \omega_{g \mid \mathfrak{m}}^{-1} \in L_{\pi(g)}(G / H) .
$$

Here we regard the elements of the fiber $L_{x}(G / H)$ as linear isomorphisms $\mathrm{m} \rightarrow T_{x} G / H . \quad \Psi$ is a principal bundle homomorphism, and $\Psi(G)$ is a $\rho(H)$ structure on $G / H$, i.e. a reduction of $L(G / H)$ to the subgroup $\rho(H)$ of $G L(\mathrm{~m})$. Since the model is of first order, $\Psi: G \rightarrow \Psi(G)$ is a bundle isomorphism (cf. [12] p. 191 or the Remark of [7], p. 193).

Now, the assumption on $\Phi$ implies that the natural torsion-free $G$-invariant linear connection of $G / H$ (cf. [7], p. 197) is reducible to $\Psi(G)$. Indeed, we recall that the $G$-invariant connections on $\Psi(G)$ are in a one to one correspondence with the linear maps

such that

$$
\Lambda: \mathfrak{m} \rightarrow \mathfrak{h}^{\prime}
$$

$$
\Lambda(\operatorname{Ad}(h) X)=\operatorname{Ad}(\rho(h)) \Lambda(X) \quad X \in \mathfrak{m}, h \in H
$$

(Theorem 2.1 of [7], p. 191). According to the assumption, we have the connection $\Gamma$ corresponding to $\Lambda:=(1 / 2) \Phi$. By using Theorem 2.10 at page 197 in [7], we see that $\Gamma$ is the natural $G$-invariant torsion free connection relative to the decomposition $\mathfrak{g}=\mathfrak{h} \oplus \mathrm{m}$. Via the bundle isomorphism $\Psi: G \rightarrow \Psi(G), \Gamma$ determines a principal $G$-invariant connection $\Gamma_{0}$ on $G \rightarrow G / H$. The horizontal space $\mathfrak{m}^{\prime}$ of $\Gamma_{0}$ at $e \in G$ is an $\operatorname{Ad}(H)$-invariant subspace of $\mathfrak{g}$ and $\mathfrak{g}=\mathfrak{h} \oplus \mathfrak{m}^{\prime}$ is a reductive decomposition. We claim that $\left[\mathrm{m}^{\prime}, \mathrm{m}^{\prime}\right] \subset \mathfrak{h}$. Indeed, $\Gamma$ coincides with the canonical $G$-invariant connection of $G / H$ with respect to the reductive decomposition $\mathfrak{g}=\mathfrak{h} \oplus \mathfrak{m}^{\prime}$ (cf. Remark on p. 193 of [7]). Thus at the point $e H$ the torsion tensor of $\Gamma$ is given by $T(Z, W)=-[Z, W]_{\mathfrak{m}^{\prime}}$. Since $\Gamma$ is torsionless we conclude that $\left[\mathfrak{m}^{\prime}, \mathfrak{m}^{\prime}\right] \subset \mathfrak{h}$. Hence a) follows.

As an application we get the following examples of reductive models without Cartan space forms modeled on them:

Corollary 6.3. Let $\mathfrak{q}$ be a compact semisimple Lie algebra. Let $\xi_{H}^{A d}(\mathfrak{g}, \mathfrak{h})$ be the standard reductive model for a Cartan geometry with group $H=\operatorname{Int}(\mathfrak{q})$. There exist no Cartan space forms modeled on $\xi_{H}^{A d}(\mathfrak{g}, \mathfrak{h})$.

Proof. The model under consideration satisfies the assumptions of the above statement. On the other hand, we showed in Theorem 4.4 that $\xi_{H}^{A d}(\mathfrak{g}, \mathfrak{h})$ is of non symmetric type.

COROllary 6.4. For each $n \in N, n \geq 3$, there exists a n-dimensional first order reductive model for a Cartan geometry such that there are no Cartan space forms modeled on it.

Proof. In the case $n=3$ it suffices to consider the standard reductive model with group $H=\operatorname{Int}(\mathfrak{q})$ where $\mathfrak{q}=\mathfrak{o}(3)$. Denote by $\xi_{H}^{A d_{0}}\left(\mathfrak{g}_{0}, \mathfrak{h}\right)$ this model. Let $n>3$; set

$$
\mathfrak{g}:=\mathfrak{g}_{0} \oplus \mathfrak{a} \quad \text { (direct sum of Lie algebras) }
$$


where $\mathfrak{a}$ is an abelian Lie algebra of dimension $n-3$. The representation $A d_{0}: H \rightarrow \operatorname{Aut}\left(\mathrm{g}_{0}\right)$ extends trivially to a representation $A d: H \rightarrow \operatorname{Aut}(\mathfrak{g})$. Hence $\xi_{H}^{A d}(\mathfrak{g}, \mathfrak{h})$ is a first order reductive model for a Cartan geometry, with group $H$, and reductive decomposition $\mathfrak{g}=\mathfrak{h} \oplus \mathfrak{m}$, where $\mathfrak{m}=\mathfrak{q} \oplus \mathfrak{a}$. Clearly, this model satisfies the assumption of Theorem 6.2. On the other hand, $\xi_{H}^{A d}(\mathfrak{g}, \mathfrak{h})$ is of non symmetric type, because otherwise there would exist a reductive decomposition $\mathfrak{g}=\mathfrak{h} \oplus \mathfrak{m}^{\prime}$ of $\mathfrak{g}$, such that $\left[\mathfrak{m}^{\prime}, \mathfrak{m}^{\prime}\right] \subset \mathfrak{h}$. This would yield a reductive decomposition of $\mathfrak{g}_{0}$ :

$$
\mathfrak{g}_{0}=\mathfrak{h} \oplus\left(\mathfrak{g}_{0} \cap \mathfrak{m}^{\prime}\right)
$$

with $\left[\mathfrak{g}_{0} \cap \mathfrak{m}^{\prime}, \mathfrak{g}_{0} \cap \mathfrak{m}^{\prime}\right] \subset \mathfrak{h}$, which is impossible since $\xi_{H}^{A d_{0}}\left(\mathfrak{g}_{0}, \mathfrak{h}\right)$ is of non symmetric type. Hence according to Theorem 6.2, there exist no Cartan space forms modeled on $\xi_{H}^{A d}(\mathfrak{g}, \mathfrak{h})$.

\section{REFERENCES}

[ 1 ] D. V. Alekseevsky, P. W. Michor: Differential Geometry of Cartan connections, Publ. Math. Debrecen 47 (1995), 349-375.

[2] D. E. BLAIR: Contact manifolds in Riemannian Geometry, Lect. Notes in Math. 509, 1976, Springer-Verlag.

[3] C. Ehresmann: Les connexions infinitesimales dans un espace fibre differentiable. Centre Belge Rech. Math., Colloque Topologie, Bruxelles, du 5 au 8 juin 1950, $29-55$ (1951).

[4] S. Kobayashi: On connections of Cartan, Can. J. Math. 8 (1956), 145-156.

[5] S. Kobayashi: Theory of Connections, Ann. Mat. Pura Appl. 43 (1957), 119-194.

[6] S. Kobayashi, K. Nomizu: Foundations of Differential Geometry, Vol. I, Interscience, NewYork, 1969.

[ 7 ] S. Kobayashi, K. Nomizu: Foundations of Differential Geometry, Vol. II, Interscience, NewYork, 1969.

[ 8 ] M. KuRANishi: CR geometry and Cartan geometry, Forum Math. 7 (1995), 147-205.

[9] A. Lotta, A. M. Pastore: The Tanaka-Webster connection for almost $\mathscr{S}$-manifolds and Cartan geometry, to appear on Arch. Math. Brno.

[10] R. I. Mizner: Almost CR structures, $f$-structures, almost product structures and associated connections, Rocky Mount. J. of Mathematics 23, no. 4 (1993), 1337-1359.

[11] J. H. Rawnsley: $f$-structures, $f$-twistor spaces and harmonic maps, in Geometry Seminar "Luigi Bianchi”" II-1984, Lect. Notes in Math. 1164, Springer-Verlag, 1985.

[12] R. W. Sharpe: Differential geometry. Cartan's generalization of Klein's Erlangen program, Graduate Texts in Mathematics 166, Springer-Verlag, New York, 1997.

[13] R. W. Sharpe: An introduction to Cartan geometries, Suppl. Rend. Circolo Mat. Palermo, Serie II 69 (2002), 61-76.

[14] N. TANAKA: On non-degenerate real hypersurfaces, graded Lie algebras and Cartan connections, Japan. J. Math. 20 (1976), 131-190.

[15] S. Tanno: Variational problems on contact Riemannian manifolds, Trans. A.M.S, Vol. 314 (1989), 349-379.

Dipartimento di Matematica-Universitì di Bari

Via E. Orabona 4

70125 BARI ITALY

e-mail: lotta@dm.uniba.it 